GA-A24863

THE GENERAL ATOMICS FUSION THEORY PROGRAM ANNUAL REPORT FOR GRANT YEAR 2004

\author{
by \\ PROJECT STAFF
}




\section{DISCLAIMER}

This report was prepared as an account of work sponsored by an agency of the United States Government. Neither the United States Government nor any agency thereof, nor any of their employees, makes any warranty, express or implied, or assumes any legal liability or responsibility for the accuracy, completeness, or usefulness of any information, apparatus, product, or process disclosed, or represents that its use would not infringe privately owned rights. Reference herein to any specific commercial product, process, or service by trade name, trademark, manufacturer, or otherwise, does not necessarily constitute or imply its endorsement, recommendation, or favoring by the United States Government or any agency thereof. The views and opinions of authors expressed herein do not necessarily state or reflect those of the United States Government or any agency thereof. 
GA-A24863

\title{
THE GENERAL ATOMICS FUSION THEORY PROGRAM ANNUAL REPORT FOR GRANT YEAR 2004
}

\author{
by \\ PROJECT STAFF
}

\author{
Work supported by \\ the U.S. Department of Energy \\ under Grant No. DE-FG03-95ER54309
}

GENERAL ATOMICS PROJECT 03726

DECEMBER 2004 


\section{ABSTRACT}

The dual objective of the fusion theory program at General Atomics (GA) is to significantly advance our scientific understanding of the physics of fusion plasmas and to support the DIII-D and other tokamak experiments. The program plan is aimed at contributing significantly to the Fusion Energy Science and the Tokamak Concept Improvement goals of the Office of Fusion Energy Sciences (OFES). 


\section{HIGHLIGHTS OF THEORY WORK IN GY04}

During the past year, considerable progress was made in each of the important areas of our research program:

- Simulations of NTM onset have led to development of a comprehensive mechanism for NTM onset in which the time dependent linear drive is a crucial element and a new understanding of the nonlinear physics mechanisms involved.

- The peeling-ballooning model for Edge Localized Modes (ELMs) was verified and now explains a wide variety of pedestal and ELM behavior from all the major tokamak experiments.

- 3-D ELM simulations using BOUT have verified aspects of the peelingballooning model in the presence of a more comprehensive physics model, and have revealed details of the early nonlinear dynamics of ELMs, including explosive radial bursts of filamentary structures.

- Simulations of ELM onset and evolution have also begun with NIMROD in a coordinated effort with BOUT simulations to describe the nonlinear physics of ELMs.

- Generalized models for active feedback of Resistive Wall Modes (RWMs) were developed and tested using several coupled codes. Plasma rotation and feedback were shown to have a mutually synergistic effect on stabilizing the RWM. This effect is most prominent when the rotation frequency approaches the critical rotation value for stabilization.

- The GYRO code has matured into a production code containing full physics needed to model real experimental discharges. A re-optimization of the collision operator was made to dramatically improve performance on the Cray-X1

- GYRO simulations of DIII-D plasmas including all the new physics match experimental data within the measurement uncertainties and reproduced the observed Bohm-scaling. Extensive global studies of DIII-D L-mode plasmas, including electromagnetic modes, collisions, profile variation and equilibrium E $\times \mathrm{B}$ sheared rotation were completed.

- A preliminary version of a new transport-timescale GYRO solver was developed. Given power flows, an iterated sequence of individual gyrokinetic simulations is used to determine the self-consistent temperature and density profiles.

- Upgrades to the GLF transport model which include using linear gyro-kinetic stability growth rates and renormalization using nonlinear gyro-kinetic 
simulations have greatly expanded the range of validity for modeling experimental discharges at high pressure and negative shear

- The Monte-Carlo Orbit-RF code has been coupled to a full-wave code and can now be applied to study the interactions between ICRF waves and neutral beam fast ions plasmas at arbitrary cyclotron harmonics

- A new formulation of the grad B and curvature pellet cloud drift model allows treatment of pellet injection and cloud drift from any poloidal launch angle. This is essential for studying and comparing results with actual experimental situations in DIII-D, where for example top vertical pellet injection or off-mid-plane pellet injection is usually done.

As a consequence of these results, scientists from the Theory Group were selected to give a number of invited talks and colloquia as highlighted in the next section. Following this are more detailed descriptions of the advances and achievements made in each of the major areas. 


\section{SIGNIFICANT PRESENTATIONS IN GY04}

- V.S. Chan gave an invited talk on "Progress and Future Directions in Confined Magnetic Fusion Simulation" at the American Physical Society April Meeting 2004, May 1-4, 2004, Denver, Colorado

- M.S. Chu gave an invited talk on "Modeling of feedback and rotation stabilization of the resistive wall mode in tokamaks" at the 45th American Physical Society Annual Meeting of Division of Plasma Physics, October 27, 2003, Albuquerque, New Mexico

- F.L. Hinton gave an invited talk on "Effects of electromagnetic turbulence in the neoclassical Ohm's law" at the 45th American Physical Society Annual Meeting of Division of Plasma Physics, October 27, 2003, Albuquerque, New Mexico

- P.B. Snyder gave an invited talk on "Extending the Peeling-Ballooning Model of ELMs: Toroidal Rotation and 3D Nonlinear Dynamics" at the 2004 Sherwood Conference in Missoula, Montana April 26-28

- P.B. Snyder will present an invited talk on "Toroidal Rotation and 3D Nonlinear Dynamics in the Peeling-Ballooning Model of ELMs" at the 2004 APS Meeting in Savannah, GA, November 15-19, 2004

- R.E. Waltz will make an oral presentation on "Advances in Comprehensive Gyrokinetic Simulations of Transport in Tokamaks" at the 2004 IAEA Fusion Energy Conference in Villamoura, Portugal, Nov.1-6, 2004. 


\section{ADVANCES IN TRANSPORT RESEARCH}

\subsection{GYRO Code Development}

Particle flow impurity flow simulations with GYRO have been done and a paper is been submitted to Physics of Plasmas. The study used GLF23 for scoping studies to look for various qualitative trends to be verified by GYRO nonlinear simulations. The GLF23 results were qualitatively verified and rather good even on a quantitative basis particularly for particle diffusion normed to ion energy diffusivity. The main results verified the existence of thermal pinches for pure plasmas with most of the electron flows coming from the trapped electrons. The pinches tend to shift to higher $\eta_{\mathrm{e}}=\mathrm{L}_{\mathrm{n}} / \mathrm{L}_{\mathrm{Te}}$ with collisions. The impurity flow studies also showed pinched flows and could be described by the D-V paradigm favored by experimental studies. Most of the study used Helium as the impurity in Deuterium. 5050 D-T mixture studies show the remarkable property that $\mathrm{D}$ and $\mathrm{T}$ have different flux rates. This will lead to a slight tendency for fuel separation away from the optimal 5050 mixture.

The neoclassical driving term and neoclassical flow diagnostics have been added to the $n=0$ GYRO gyrokinetic equations. In addition we have added ion-ion collisions as a number, energy, and momentum conserving Krook operator and an electron on ion drag to go with the electron ion pitch angle scattering. We recovered the neoclassical parallel ion flow velocity and bootstrap current. The parallel ion flow requires a very long time to build up from zero, This delay can be circumvented by starting with the flow in the initial condition. We were able to recover the standard neoclassical flows in the limit of vanishing $\rho^{*}$ as well as large orbit finite $\rho^{*}$ with profiles, including ambipolar ion radial particle flows. We have succeeded in showing that as expected the neoclassical driver has little effect the $\mathrm{E} \times \mathrm{B}$ turbulent flows apart from providing some parallel flow shear drive via the $\mathrm{E} \times \mathrm{B}$ nonlinearity (normally accounted for by modifications to the $\omega_{*}$ drive). The ion-ion collisions give a small damping to the zonal flows increasing the $\mathrm{E} \times \mathrm{B}$ high-n transport only slightly as expected. Furthermore there is no evidence that the zonal flows modify the standard (or large orbit) neoclassical flows when averaged over a few gyroradii. Thus it appears standard neoclassical flows can be safely added to turbulent flows as conventionally assumed, i.e. there is no significant "cross-talk" between turbulent and neoclassical flows.

During the past year we have developed feedback methods in GYRO to obtain simulations at constant (given) power and particle flows. The conventional simulations are done with fixed gradients (given experimental profiles). We use a diagonal feedback relation between flows and gradients to tune the gradients as the simulation goes forward 
so that the simulated flows match the given (experimental) flows. We can then integrate the gradients away from a pivot point of fixed temperature and density, obtain the transport solution profiles to be compared with experiment. Since core transport is very stiff, this is the only satisfactory way to compare realistic simulations to experiment. We believe this is the first step to a gyrokinetic steady state transport code. Figure 1 gives a flux-tube illustration.
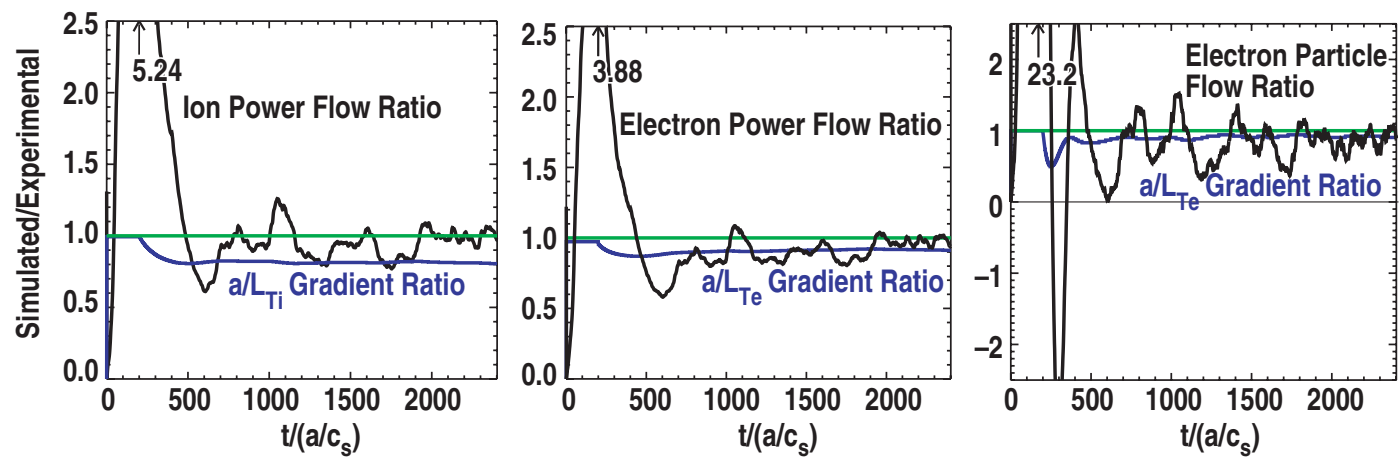

Fig. 1. Simulated / Experimental flow (black) and gradient(blue) ratios versus time for the DIII-D $\rho_{*}=0.004 \mathrm{r} / \mathrm{a}=0.6$ parameters. The case has experimental ExB shear but otherwise flat profiles for an $80 \rho_{s}$ slice with $16 \mathrm{n}$-modes, $\beta=0$ and $\sqrt{m_{i} / m_{e}}=20$. Feedback adjustment of gradients starts at $\mathrm{t}=200$.

\subsection{GLF23 Transport Modeling and Code Development}

A new closure scheme for the six-moment gyro-fluid equations for the circulating particles used in GLF2003 was developed. This scheme uses a time-independent differential equation to close the highest moments and allows a better fit to the variation of the kinetic response function with parallel wave number. The new closure and minimization scheme gives a similar accuracy for the density response $(2.7 \%)$ but is much more accurate for the higher moments ( $4.5 \%$ for pressure). The differential equation for the closure can be easily solved in the Hermite basis representation used in the new GLF2003 code and is extendable to finite trapped fraction for the circulating particles.

The development of a new set of gyro-Landau Fluid (GLF) equations, which provide a more accurate approximation to the gyro-kinetic linear stability eigenmodes has been recently finished. These equations will be the basis for the next generation of the quasilinear transport model GLF23. The new equations reduce to the six moment GLF equations of Beer and Hammett when there are no trapped particles. With finite trapped fraction there are three equations for the trapped particles and twelve for the circulating particles. The doubling of the number of equations for the circulating particles is needed in order to accurately represent the subtraction of the trapped particle region integration 
from the circulating particle density. Taking moments over the trapped region of velocity space requires new Maxwellian weighted integrals that replace the familiar modified Bessel functions (finite Lamor radius terms) in the moment equations. These integrals have been fit with rational function approximations for quick numerical calculation. The closure coefficients for the Landau damping and the curvature drift terms become functions of the trapped particle fraction. These fitted coefficients have been tabulated for interpolation to arbitrary trapped fraction. It was discovered that the zero frequency point for the perpendicular temperature moment was not correct at finite perpendicular wavenumber in all previous GLF systems. This was due to the neglect of some finite Lamor radius terms. The new system of equations is valid for all aspect ratios all trapped fractions and all perpendicular wavenumbers. The linear growth rates and frequencies from the new GLF system have been benchmarked with a very large database of gyrokinetic results. This database includes the plasma condition in the mid-radius of L-modes and H-modes for which the GLF23 equations gave good results. In addition parameter scans around conditions typical of the top of the H-mode pedestal (strong magnetic shear and strong Shafranov shift) and in the steep gradient barrier region of a negative central shear discharge have been added. The new GLF equations give an excellent approximation to the gyro-kinetic results over the whole database. The new equations can also seamlessly transition from ion temperature gradient modes to trapped electron modes to electron temperature gradient modes as the poloidal wavenumber increases. A paper on the derivation of the new GLF equations and its testing as a linear eigemode solver against the gyro-kinetic database is in preparation.

Nonlinear scans in $\mathrm{E} \times \mathrm{B}$ shear with and without the destabilizing effects of parallel velocity shear have been carried out using the GYRO gyrokinetic code including kinetic electrons. Without parallel velocity shear, the electron and ion thermal transport is quenched in the vicinity of $\gamma_{\mathrm{E}} / \gamma_{\max }=1$.92.0. When the parallel velocity shear is included, the transport is not quenched by $\mathrm{E} \times \mathrm{B}$ shear (Fig. 2). As shown in the Fig. 2, transport is strongly suppressed for $\mathrm{E} \times \mathrm{B}$ shear rates up to $50 \%$ of $\gamma_{\max }$. Beyond $\gamma_{\mathrm{E}} / \gamma_{\max }=0.5$, the transport becomes weakly sensitive to $E \times B$ shear. Here, the parallel velocity shear rate $\gamma_{P}$ is varied along with $\gamma_{E}$ using the expression $\gamma_{P}=(R q / a) \gamma_{E}$ with $R / a=6$ and $q=2$. The same simulations assuming adiabatic electrons are in the process of being completed. Both scans will serve as important benchmarks for the next generation version of the GLF23 transport model. 


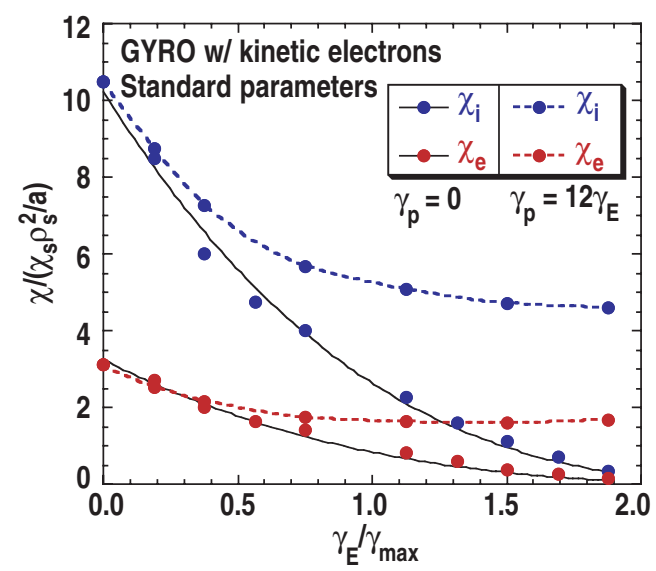

Fig. 2. Comparison of ion and electron thermal diffusivities as a function of ExB shear computed using GYRO with (dashed curves) and without (solid curves) the destabilizing effects due to the parallel velocity shear.

\subsection{ONETWO 1-1/2 D Transport Code Development}

An interface to integrate the equilibrium code TOQ into the ONETWO transport code has been developed. Specific inconsistencies in the calculations of equilibria between the existing ONETWO equilibrium solver and TOQ have been eliminated. Details in the calculation of the metrics are being worked out to create a consistent and reliable interface. TOQ is based on a flux coordinate system and can provide more accurate metric coefficients for use with ONETWO.

The NTCC based fast ion Monte Carlo package NUBEAM derived from TRANSP is now fully incorporated into ONETWO. Our final installation differs significantly from the original attempt in that NUBEAM is now a separate module in ONETWO. The latter approach became possible after a new release of a driver by the Princeton/Lehigh team. Since it is impractical to treat turbulent confinement models and fast ion slowing down calculations on the same time base, allowance was made in ONETWO to use large time steps in NUBEAM. The associated thermal ion and energy sources are obtained by a combination of extrapolation and interpolation in time.

A new TORAY-GA version 1.7 is ready to be released to the public. Various improvements have been made to this new version, including a more flexible allocation of equilibrium and radial grid points. A comprehensive testing procedure to ensure the robustness and reproducibility of TORAY-GA across platforms using a test suite has also been established.

\subsection{Neoclassical Transport in Small and Large Rotation Limit}

A formulation of the neoclassical transport theory for tokamak plasmas with large toroidal velocities that can be comparable to the ion thermal velocity has been developed 
using the drift kinetic equation. In this formulation, the motion of the guiding centers is the same as in the small rotation theory, but the radial electric field is considered stronger: the ordering $E_{\mathrm{r}} / \mathrm{B}_{\theta} \sim \mathrm{v}_{\mathrm{i}}$ is adopted where $\mathrm{B}_{\theta}$ is the poloidal magnetic field and $v_{i}$ is the ion thermal velocity. A complete set of transport coefficients for both electrons and ions is calculated in the large-aspect-ratio limit in the banana regime for flux surfaces of arbitrary shape. The calculation utilizes a recently developed method of matched asymptotic expansions for particle and energy fluxes and a regular perturbation for the angular momentum flux. The consistent application of perturbation for large-aspect-ratio provides a satisfactory explanation why the angular momentum flux is much smaller than the ion energy flux. 


\section{ADVANCES IN MHD STABILITY RESEARCH}

\subsection{Edge Stability, ELM Onset, and Pedestal Constraints}

A study of large and small ELM discharges from the JET-DIII-D comparison experiment was carried out using ELITE. Both discharges were found to be near the peeling-ballooning stability boundary when ELMs were observed, as expected from the peeling-ballooning model. An $n=11$ mode was found to be most unstable for the small ELM case with a narrower density pedestal, while a wider $n=8$ mode was most unstable for the large ELM case.

The first detailed studies of toroidal shear effects on intermediate to high $\mathrm{n}$ modes have been carried out using a new formulation, implemented in the ELITE code. Growth rate and mode structure as a function of toroidal rotation rate were studied in DIII-D shot 113207, using measured density and temperature profiles and an EFIT equilibrium reconstruction with bootstrap current at a time immediately preceding an ELM. Toroidal rotation, at measured values, was found to significantly alter the calculated mode structure, causing mode narrowing and leading to changes of sign in the radial phase. This is shown in Fig. 3. Growth rates were modified only slightly, with less than a $20 \%$ change even for twice the measured rotation. However, rotation can have a strongly stabilizing effect at low magnetic shear, and more generally for high $\mathrm{n}(\mathrm{n}>30)$ modes.
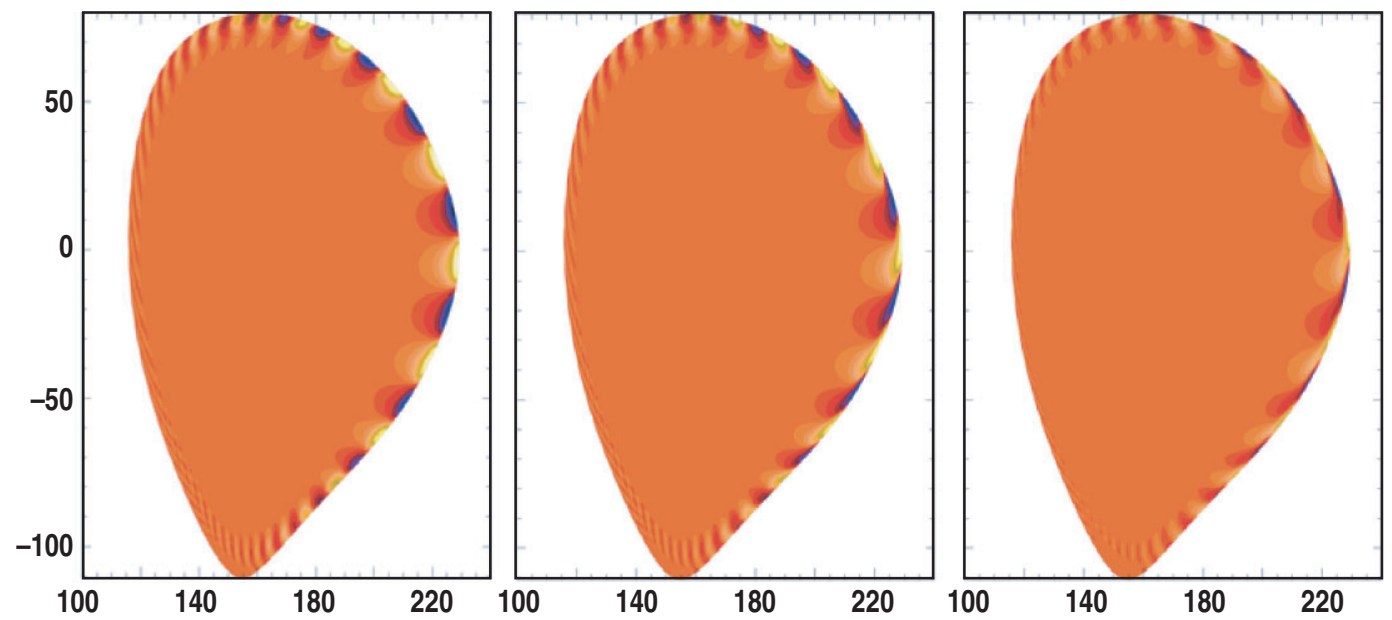

Fig. 3. Mode structure of the most unstable mode $(n=11)$ just prior to an ELM in DIII-D shot 113207 , calculated by ELITE with (a) no rotation, (b) the measured pedestal rotation of $4 \mathrm{kHz}$, (c) rotation of $10 \mathrm{kHz}$. The calculated growth range changes only a small amount, but the mode structure and radial phase are altered significantly by toroidal rotation shear. 
A detailed study of the stability of AUG type II ELM discharges has been carried out using model equilibria based on parameters provided by IPP Garching. The study found that for type II parameters of high density and strong shaping, edge stability is limited by high $n$ pure ballooning modes, while for lower densities where larger ELMs are observed, intermediate $n$ peeling-ballooning modes are the limiting instability. The results are consistent with the peeling-ballooning model of ELMs.

Recent studies on DIII-D using edge current profiles measured by the new lithium beam diagnostic find edge current profiles similar to those given by bootstrap current models, and, as in previous studies using model current profiles, intermediate $\mathrm{n}$ peelingballooning modes are found to go unstable shortly before ELM onset.

\subsection{ELM Dynamics}

In collaboration with LLNL, an investigation of the nonlinear dynamics of ELM-like peeling-ballooning modes with the BOUT 3D reduced Braginskii simulation code is continuing. We have conducted new sets of simulations employing a zero electrostatic potential boundary condition at the outer boundary. These simulations clearly reveal rapid onset of large-scale instabilities on typical peeling-ballooning timescales for unstable equilibria. Reducing the edge gradients eliminates these fast growing modes, as expected from linear peeling-ballooning theory, and consistent with these fast growing edge instabilities providing the mechanism for ELMs. Observed wavelengths and structure in the linear phase of the simulations are consistent with expectations from ELITE calculations. The simulations have been successfully carried into the early nonlinear phase, with the observation of poloidal and toroidal narrowing and fast outward radial bursts of filaments of particles from near the top of the pedestal out into the scape off layer. A number of similarities to dynamic ELM observations on DIII-D and MAST are seen in the simulations.

Work is continued on a benchmarking study using ELITE and the NIMROD 3D MHD code. An edge localized mode, similar to that found by ELITE, was found with NIMROD. Growth rates within a factor of two of ELITE's were obtained by modifying the resistivity profile used in NIMROD.

Work to model ELMs using the 3-D MHD code NIMROD is continued. The nonlinear phase of the ELM evolution shows many of the properties observed in the BOUT simulations, such as peaking of the mode structure off the outboard midplane axis, and the filamentation of the plasma density and temperature moving outward due to counter rotating eddy pairs (Fig. 4). In these simulations, the edge transition region has been made much sharper than the one used in the previous study and only toroidal modes $n=0-21$ are included. The equilibrium chosen for this study gives a spectrum of linear growth rates which plateaus near $n=10$. 

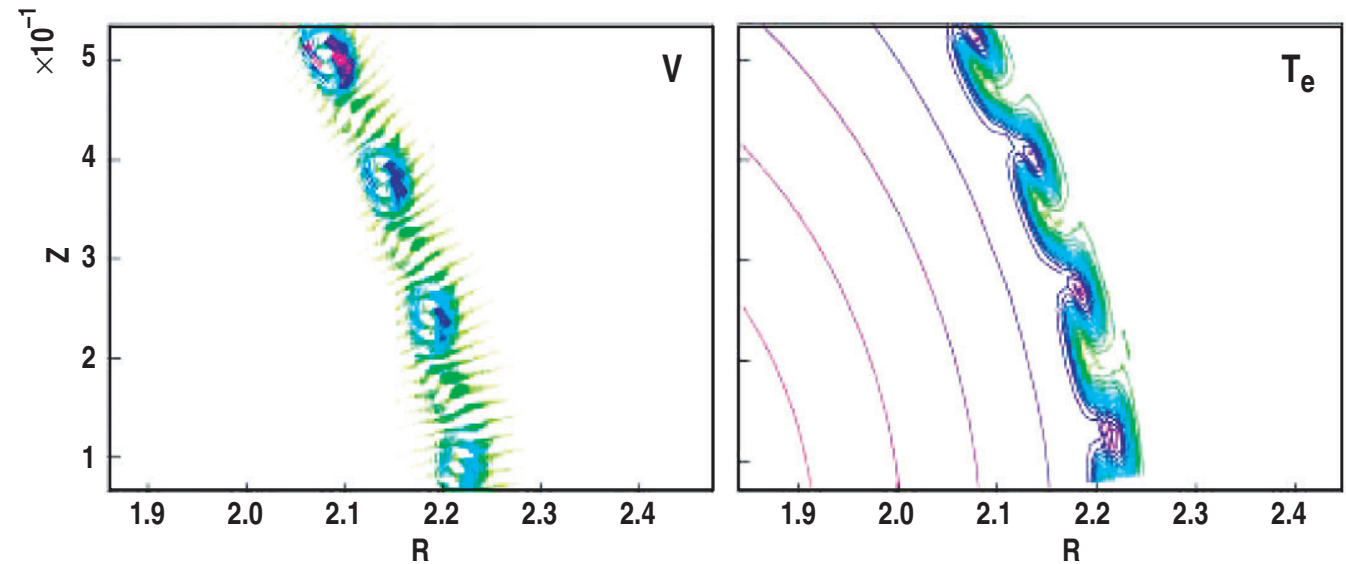

Fig. 4 Velocity vectors and electron temperature in the upper outboard region during the early nonlinear phase of an ELM simulation using NIMROD.

\subsection{Rotation and Feedback Stabilization of Resistive Wall Mode}

The characteristics of feedback stabilization of the resistive wall mode (RWM) in DIII-D geometry are studied systematically using the MARS-F stability code for a set of equilibria with $\beta_{\mathrm{N}}$ varying from the no wall ideal limit $\beta_{\mathrm{N}}{ }^{\mathrm{NW}}$ to the ideal wall limit $\beta_{\mathrm{N}}{ }^{\mathrm{IW}}$, and with experimentally measured characteristics of the amplifier circuit. The upper limit in $C_{\beta}=\left(\beta_{N}-\beta_{N}{ }^{N W}\right) /\left(\beta_{N}{ }^{I W}-\beta_{N}{ }^{N W}\right)$ is related to the inherent phase lag introduced by the amplifier circuit and to a lesser extent introduced by the time delay. The frequency range characteristics of the feedback need to be extended by more than a factor of 10-12 before an equilibrium with $\mathrm{C}_{\beta}=92 \%$ can be stabilized with a P-controller.

Ideal calculations for a sequence of fully bootstrapped equilibria obtained by scaling the vacuum toroidal field show instability with no wall but stability with the DIII-D wall and with considerable structure in the growth rates as functions of $\mathrm{q}_{0}, \mathrm{q}_{\mathrm{min}}$, and qedge. Minima and maxima in the growth rates do not correspond to integer values of any of $\mathrm{q}_{0}$, $\mathrm{q}_{\mathrm{min}}$, and qedge. This result implies that active feedback control of the associated RWM should also be quite insensitive to these parameters, which may simplify feedback schemes.

\subsection{Non-linear Tearing Mode Analysis}

Work to study the effects of sawtooth seeding on $3 / 2$ NTMs using NIMROD is continued. The effects of rotational shear on the evolution of the $3 / 2$ tearing modes are being investigated using model TOQ equilibria with various rotational profiles, and EFIT DIII-D equilibria with experimental rotation profiles. The kinetic energy of the nonlinearly driven mode shows interesting behavior suggestive of propagation in the fluid frame, and perhaps the theoretically expected spin down from the rotational frequency of the $1 / 1$ mode. 
A series of NIMROD non-linear simulations to investigate the role of linear instability drive in the NTM seeding in Alcator C-Mod discharges has been carried out based on experimentally reconstructed equilibria. The theory developed for the onset and early evolution of NTMs in sawtoothing DIII-D discharges appear to be also valid for CMod. Preliminary C-Mod results are consistent with the importance of both the classical linear and the nonlinear coupling drive to the tearing mode onset and early evolution.

The effects of varying rates of heating on the spontaneous onset of tearing modes in tokamaks have also been simulated with NIMROD. Here the heating is implemented by increasing the core pressure with time, using a DIII-D equilibrium as an initial condition. The 2/1 tearing mode is driven unstable and the results agree with 2003 published results from island evolution modeling of a DIII-D experiment. The island width as a function of beta is a specific function of the rate of heating. Efforts continue to simulate nonlinear saturation at small island size for low heating rates.

A re-analysis of published simulation predictions for the early MHD bursts in the $\beta_{\mathrm{N}}$ $\sim 2$ L-mode NCS discharge 87009 shows that many of the numerical predictions are exhibited in more recent, lower $\beta_{\mathrm{N}}$ L-mode NCS discharges. In both the simulations using the NFTC and FAR resistive MHD codes and the experiments, the initially localized resistive interchange mode at the inner rational surface broadens until it reaches the magnetic axis. This reconnects either at, or just off-axis, consistent with a double tearing structure. The inner island then dissipates forming a single tearing mode.

\subsection{DIII-D Stability Analysis}

Investigations into the ideal MHD stability of tokamaks with a current hole region suggest that their stability can be treated as a plasma with an internal "vacuum like" region. For perturbations with finite toroidal mode number, the current hole region behaves exactly like a vacuum region. This is consistent with standard tokamak external kink analyses. For axisymmetric perturbations, however, the analysis is more subtle. The minimizing perturbation consists of the superposition of three different kinds of independent displacements: a vertical displacement and two other displacements that compress the plasma and the toroidal magnetic field, respectively.

The two fluid effects on the onset of a $2 / 1$ tearing mode in DIII-D are being investigated. Accurate EFIT equilibrium reconstructions leading up to the onset of the 2/1 mode were used in the analysis. The solution for $\Delta^{\prime}$, evalutated using the PEST-III code, was matched to an inner layer solution using the Glasser, Greene and Johnson model to get a reasonable MHD growth rate for the linear 2/1 mode. A critical positive value of $\Delta^{\prime}$ necessary for onset comes out of the analysis, and agrees with experimental onset point. The two fluid dispersion relation, which depends on the resistive MHD growth rate, suggests that the two fluid effects are important in the evolution but require linear MHD instability for mode growth. 
In an effort to resolve the discrepancy in the vertical separatrix locations between magnetic and Thomson scattering measurements observed in some DIII-D discharges, an experiment was performed to investigate the effects of 3-D error fields on magnetic surfaces using the I-Coil. Slowly rotating $n=1$ traveling waves at $5 \mathrm{~Hz}$ and various amplitudes $(\sim 0.1-0.3 \%$ of the poloidal equilibrium field $)$ were applied to perturb the edge magnetic surfaces by pre-programming the I-Coil currents. At $0.1 \%$ perturbation, the vertical separatrix location difference between magnetic reconstructions which assume toroidal symmetry and Thomson scattering measurements of $\mathrm{T}_{\mathrm{e}}$ responds in phase to the applied perturbed field with an oscillation amplitude $\sim 2 \mathrm{~cm}$. At $0.3 \%$ perturbation, the amplitude of difference in the separatrix location grows in time leading to an early discharge termination due to appearance of a locked mode. The results are consistent with the conjecture that the observed separatrix location differences between magnetic and Thomson scattering measurements are due to the small toroidal asymmetry of the external shaping coil locations.

\subsection{Resistive Linear Stability Code (TWIST-R) Development}

The resistive MHD energy principle is being implemented in the TWIST-R code using the new algorithm for solving differential equations with singular solutions. The new algorithm is also appropriate for solving the inner layer equations required for matching to the external region TWIST-R solutions. Considerable progress was obtained over the past year. The serious unphysical behavior in nonresonant harmonics near the magnetic axis was eliminated. The 2D TWIST-R code now reproduces the main features of the PEST-III solution except for some remaining unphysical behavior in nonresonant harmonics near the magnetic axis, which, however, does not appear to affect the solution near the resonant surface. 


\section{Advances in RF Heating and Fueling Research}

\subsection{ICRF and NBI Fast lon Interactions}

The TORIC full wave code has been selected for coupling with the ORBIT-RF ICRF orbit code after careful review. TORIC solves the finite Larmor radius wave equations in the ion cyclotron range of frequencies in arbitrary toroidal geometry. TORIC is solved using DIII-D equilibrium data to provide the radial profiles of left $\left(\left|\mathrm{E}_{+}\right|\right)$, right $\left(\left|\mathrm{E}_{-}\right|\right)$ polarized components, $\mathrm{n}_{/ /}$component and the solution of dispersion relation $\left(n_{\perp}^{2}\right)$ of fast wave along the equatorial plane. All data are stored in a table format as a function of poloidal mode number $(\mathrm{M})$ and radial position $(\mathrm{R}, \mathrm{Z}=0)$. TORIC produces the magnitudes of $\left|E_{+}\right|$and $|E|$ in $V / m$ for 1 Amp antenna current, which can then be scaled to a given input power in principle if the antenna current is known from experiment. Since this information is usually not available, an alternate approach is to scale $\left|E_{+}\right|$and $\left|E_{-}\right|$using the ratio of the square root of the total power absorption from experiments and that from TORIC simulation using an antenna current of $1 \mathrm{~A}$. These renormalized data are passed on to ORBIT-RF to calculate the increase in perpendicular energy of particles passing through the resonance surfaces and thus the absorbed power. To evaluate the power absorption when the ion distribution has evolved to a steady state, the calculated power that goes to the energetic ions has to be the same as the power transferred by the energetic ions to the background. That has been verified.

\subsection{Pellet Fueling Analysis}

The $\nabla \mathrm{B}$ induced pellet cloud drift model was generalized to include new physical effects 1) enhanced curvature drive associated with near-sonic parallel flows; 2) incorporation of realistic (non flat) plasma pressure profiles; 3) helical magnetic field line geometry (rotational transform); and 4) "mass shedding" caused by the disabling effect of magnetic shear on the coherency of the $E / B$ cloud drift. These new effects have not been addressed in previous theoretical models, The new formulation allows us to treat pellet injection and cloud drift from any poloidal launch angle. Previously, the model was restricted to only mid-plane HFS or LFS pellet launches. This new work is essential for studying and comparing results with actual experimental situations in DIII-D, where for example top vertical pellet injection or off-mid-plane pellet injection is usually done. This had led to interesting results. We found that the cloud can have a bulk poloidal drift as well as the drift in minor radius (across flux surfaces). For pure midplane HFS or LFS launch the poloidal drift is zero. But for purely vertical launch (top or bottom) the cloud 
drift is predominately poloidal. However, it will acquire a small positive minor radius drift because the main poloidal drift moves the cloud over to the LFS of the magnetic axis (never the HFS unfortunately). That is the reason for the radial drift in the outward (unfavorable direction). However, the outward drift is still not as bad as injecting the pellet from the LFS mid-plane position where the outward drift is much more pronounced. Theoretical deposition profiles $\Delta n$ for midplane outer wall $\chi_{c 0}(0)=0$, and above midplane inner wall $\chi_{c 0}(0) \sim 3 \pi / 4$ pellet injection cases on DIII-D are shown in Figs. 5 and 6 respectively. The electron density perturbation $\Delta n$ measured by Thomson scattering less than $1 \mathrm{~ms}$ after the ablation process is overlaid. We see that the present model compares well with measured profiles in both cases. It should be mentioned that in the outer wall injection case, the theory and experimental profiles deviate sharply for $\rho / a$ $>\sim 0.85$. This is likely due to a strong edge localized mode (ELM) triggered during the pellet injection event, which causes a large fraction of the edge pedestal density to be ejected from the plasma. This is also consistent with the modest discrepancy between theoretical and experimental particle fueling efficiencies, $66 \%$ versus $46 \%$, respectively. For inner wall injection the triggered ELM is much less pronounced-both measured and calculated fueling efficiencies are nearly $100 \%$.

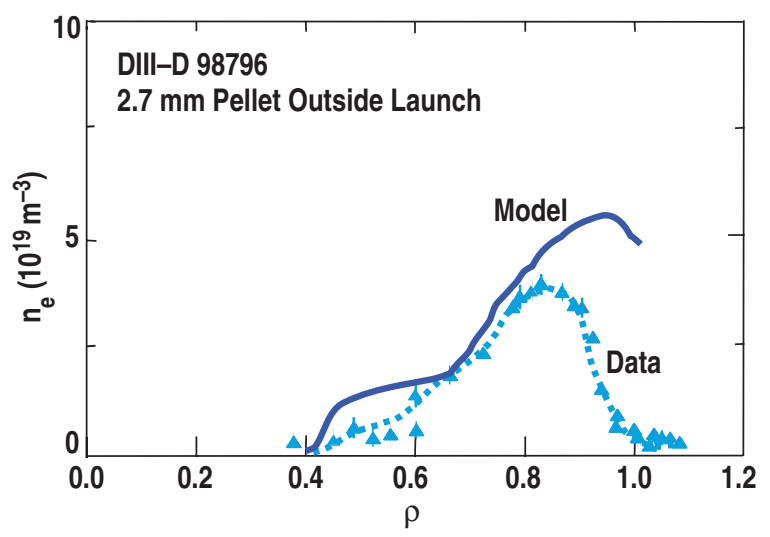

Fig. 5. Measured pellet mass deposition for a $2.7 \mathrm{~mm}$ cylindrical $\mathrm{D}_{2}$ pellet (equivalent spherical radius $1.54 \mathrm{~mm}$ ) injected at $586 \mathrm{~m} / \mathrm{s}$ from the outside midplane, $\chi_{c 0}(0)=0$, in an ELMing H-mode plasma overlaid with the modeling result using measured profiles and pellet parameters. 


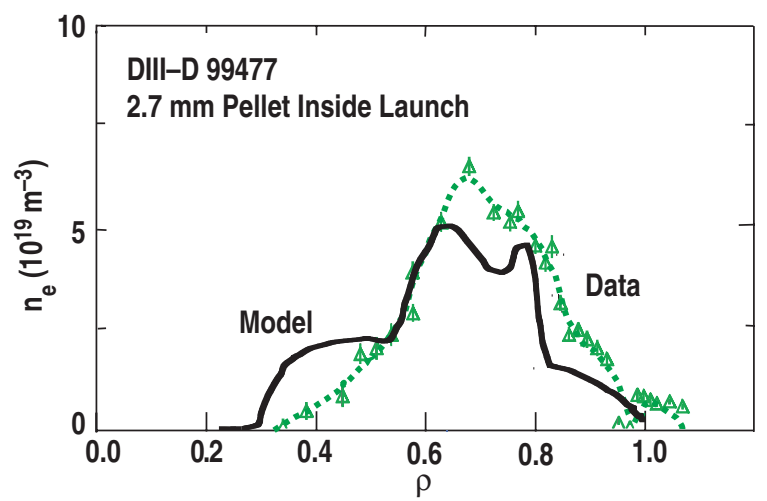

Fig. 6. Measured pellet mass deposition a $2.7 \mathrm{~mm}$ cylindrical $\mathrm{D}_{2}$ pellet injected at $153 \mathrm{~m} / \mathrm{s}$ from the inside wall $35 \mathrm{~cm}$ above the midplane, $\chi_{c 0}(0) \sim 3 \pi / 4$, in an ELMing H-mode plasma overlaid with the modeling result using measured profiles and pellet parameters. 


\section{ADVANCES IN INNOVATIVE CONFINEMENT CONCEPT RESEARCH}

\subsection{Magnetic Target Fusion}

This year we further examined the magnetic target fusion concept using a spherically symmetric imploding plasma liner formed by merging an array of focused plasma jets. The liner is supposed to crush a magnetized plasmoid, and heat it to ignition conditions. The advantage of using jets is that they provide good standoff distance between the energy source and the implosion site. In the previous year, our theoretical model showed that ignition required ultra-supersonic jets $(M>50)$. However, the model assumed, naively, that the merged jets would form a nearly homogeneous plasma liner with the same $M$ number as the individual jets.

This simple picture changed. Instead, we came to the realization that when the jets collide with each other at a very oblique angle they under go a sudden but slight change in their direction, which can only be accomplished by an oblique shock. This is analogous to the supersonic flow past a wedge with a small half-angle, where the flow is refracted across an oblique shock. Surrounding each jet is a narrow hexagonal boundary layer containing the hot shocked material. After the merging phase is completed the newly formed shocked layer is no longer in static pressure equilibrium. Consequently, in the process of breaking up it exchanges heat with the cool un-shocked interior. Equipartition of heat downgrades the liner Mach number from 50 to $\sim 9$. Thus shock heating puts the liner on a high adiabat, degrading its effectiveness as a pusher. A more refined examination to confirm this result is underway.

\subsection{3-D Equilibrium Reconstruction}

Various linearization schemes to approximate the signal gradients and to accelerate the search for the optimum state vector (plasma equilibrium) based on the efficient 2-D EFIT optimization scheme have been formulated for use with the 3-D equilibrium reconstruction code V3FIT. The two response function and magnetic signal codes V3RFUN and V3POST have been incorporated into the 3-D stellarator optimization code STELLOPT to provide a prototype 3-D reconstruction code that can be used to examine various numerical features of the reconstruction process, such as the level of convergence needed to estimate the signal gradients, and to guide the development of the optimized V3FIT code. 


\section{PUBLICATIONS}

\subsection{Primary Theory Authors}

1. Candy, J., Waltz, R.E., Dorland, W., "The local limit of gyrokinetic simulations", Phys. Plasmas 11 (2004) L25.

2. Candy, J., Waltz, R.E. and Rosenbluth, M.N. "Smoothness of turbulent transport across a minimum q-surface", Phys. Plasmas 11 (2004) 1879.

3. Chu, M.S., A. Bondeson, M.S. Chance, Y.Q. Liu, A.M. Garofalo, A.H. Glasser, G.L. Jackson, R.J. La Haye, L.L. Lao, G.A. Navratil, M. Okayabashi, H. Reimerdes, J.T. Scoville, and E.J. Strait "Modeling of feedback and rotation stabilization of the resistive wall mode in tokamaks" Phys. Plasmas, Vol. 11, p.2497 (2004).

4. Chu, M.S. and P.B. Parks, "Minimizing the MHD Potential Energy for the Current Hole Region in Tokamaks" to appear in Physics of Plasmas, Vol.11 (2004).

5. Estrada-Mila, C., Candy, J., Waltz, R.E. , "Gyrokinetic Simulations of Ion and Impurity Transport", to be submitted to Phys. Plasmas.

6. Guo, S.C. and M.S. Chu, "Nonlinear tearing mode interactions and mode walllocking in RFP plasmas" Physics of Plasmas, Vol.11, p. 4050 (2004).

7. Hinton, F.L., Waltz, R.E., Candy, J, "Effects of electromagnetic turbulence in the neoclassical Ohm's law" Phys. Plasmas 11 (2004), 2594.

8. Hirshman, S.P., E.A. Lazarus, J.D. Hanson, S.P. Knowlton, L.L. Lao, "Magnetic Diagnostic Responses for Compact Stellarators," Phys. Plasmas 11, 595 (2004).

9. Hollmann, E.M., Groth, Jernigan, T.C., Parks, P.B., et al. "Induced Radiative Collapse with Noble Gas Injectio in the DIII-D Tokamak" to be submitted to Nuclear Fusion, 2004.

10. Kinsey, J.E., "GLF23 modeling of turbulent transport in DIII-D," to be submitted to Fusion Science and Technology (2004), General Atomics Report GA-A23641 to be printed.

11. Kinsey, J.E. G.M. Staebler, and R.E. Waltz, "Predicting Core and Edge Transport Barriers in Tokamaks Using the GLF23 Driftwave Transport Model," submitted to Phys. Plasmas (2004).

12. Kinsey, J.E., R.E. Waltz, and J. Candy, "Nonlinear Gyrokinetic Simulations of ExB Shear Quenching of Transport," submitted to Phys. Plasmas (2004). 
13. Parks, P.B., R. Ishizaki, N. Nakajima, M. Okamoto, “Two-Dimensional Simulation of Pellet Ablation With Atomic Processes,” Phys. Plasmas 114064 (2004).

14. Parks, P.B. and Baylor, L.R. "Effect of parallel Flows and toroidicity on cross-field transport of pellet ablation matter in tokamaks" GA-A24807 submitted to Phys. Rev. Lett., September 2004.

15. Petty, C.C., T.C. Luce, D.C. McDonald, J, Mandrekas, M.R. Wade, J. Candy, J.G. Cordey, V. Drozdov., T.E. Evans, J.R. Ferron, R. J. Groebner, A.W. Hyatt, G.L. Jackson, R.J. LaHaye, T.H. Osborne, R.E. Waltz, "Beta Scaling of Transport on DIII-D Tokamak: is Transport Electrostatic or Electromagnetic?" to be published in Physics of Plasmas, May 2004.

16. Rewoldt, G., J.E. Kinsey, "Comparison of Linear Microinstability Calculations of Varying Input Realism," Phys. Plasmas 11844 (2004).

17. Snyder, P.B., H.R. Wilson, J.R. Ferron, L.L. Lao, A.W. Leonard, D. Mossessian, M. Murakami, T.H. Osborne, A.D. Turnbull, X.Q. Xu, "ELMs and Constraints on the H-Mode Pedestal: A Model Based on Peeling-Ballooning Modes," Nucl. Fusion 44, 320 (2004).

18. Snyder, P.B., H.R. Wilson, T.H. Osborne, and A.W. Leonard, "Characterization of Peeling-Ballooning Constraints on the Pedestal in Tokamaks", Plasma Phys. Control. Fusion 46 A131 (2004.

19. Staebler, G.M., "Poloidal Momentum Transport due to Turbulence in Toroidal Geometry,” Phys. Plasmas 111064 (2004).

20. Turnbull, A.D., P.B. Snyder, D.P. Brennan, M.S. Chu, L.L. Lao, "Magnetohydrodynamics Theory and Simulation Basis for DIII-D," to be submitted to Fusion Science and Technology (2004); General Atomics Report GA-A24627 to be printed.

21. Waltz, R.E., " $\rho$ *-Scaling and Physically Realistic Gyrokinetic Simulations of Transport in DIII-D," to be submitted to Fusion Science and Technology (2004); General Atomics Report GA-A24618 to be printed.

22. Wong, S.K. and V.S. Chan, "A Drift-Kinetic Approach to Neoclassical Transport Theory for Plasmas with Large Toroidal Rotation”, Phys. Plasmas 113432 (2004). 


\subsection{Presentations at Conferences and Workshops}

1. Dylan Brennan presented "Dynamic Equilibirum Effects in Tearing Mode Onset and Nonlinear Evolution" at APS DPP03 in Albuquerque, New Mexico.

2. Dylan Brennan presented "Comparisons of Predicted Quasi-Static Nonlinear States of Tearing Modes" at the Sherwood Fusion Theory conference Missoula, Montana, April 26-28, 2004.

3. J. Candy gave an invited presentation "Recent Gyrokinetic Simulations with GYRO: Bohm Transport in DIII-D, the Local Limit of Global Simulations, and Transport Across a Minimum-q Surface" at the JIFT Theory-Based Modeling and Integrated Simulation of Burning Plasmas Workshop and 21 COE Workshop on Plasma Theory at Kyoto University, Japan Dec 15-17, 2003.

4. V. Chan, gave a talk on "Theory and experiment of electron cyclotron current drive on DIII-D", at the 2003 Asian Plasma and Fusion Association Meeting, October 13-16, 2003, Hangzhou, China.

5. V. Chan gave a talk on "Modeling of Neutral Beam Ion Absorption of High Harmonic ICRF Waves in the DIII-D Tokamak" at the JIFT Theory-Based Modeling and Integrated Simulation of Burning Plasmas Workshop and $21 \mathrm{COE}$ Workshop on Plasma Theory at Kyoto University, Japan Dec 15-17, 2003.

6. S.C. Chiu, "Estimates of Transport Near the Tokamak Axis", Bull. Am. Phys. Soc. 48, 260 (2003).

7. M. Choi, "Neutral Beam Ion Absorption of High Harmonic ICRF Waves in the DIII-D Tokamak", Bull. Am. Phys. Soc. 48, 267 (2003).

8. C. Estrada-Mila, "GYRO Study of Turbulent Transport of Impurities", Bull. Am. Phys. Soc. 48, 261 (2003).

9. C. Estrada-Mila, "Gyrokinetic Simulations of Plasma Transport and Isotope Effects" presented at the 2004 Sherwood Conference in Missoula, Montana April 26-28.

10 F.L. Hinton, "Electromagnetic Turbulence Modification of Neoclassical Transport" presented at the 2004 Sherwood Conference in Missoula, Montana April 26-28.

11. J. Kinsey, "Recent Advanced in Predicting Core and Edge Barriers Using the GLF23 Transport Model”, Bull. Am. Phys. Soc. 48, 261 (2003).

12. Jon Kinsey presented "GLF23 Modeling of Core and Edge Barriers," at the Sixth Meeting of the ITPA Confinement Database and Modeling and Transport Physics Topical Groups, Naka, Japan, Mar. 8-11, 2004.

13. L. Lao, "Modernization and Improved Capabilities, and 3D Extension of the EFIT Equilibrium Code”, Bull. Am. Phys. Soc. 48, 264 (2003).

14. P. Parks, "Refueling Fusion Plasmas by a High-Energy-Density Jet", Bull. Am. Phys. Soc. 48, 258 (2003). 
15.P.B. Parks, "Effect of Toroidicity on Fast Fuel Relocation in Tokamaks" presented at the 2004 Sherwood Conference in Missoula, Montana April 26-28.

16. P. Parks presented "Recent Advances in the Theory and Simulation of Pellet Ablation and Fast Fuel Relocation in Tokamaks" at the 2004 IAEA Fusion Energy Conference in Villamoura, Portugal, Nov.1-6, 2004.

17.Pomphrey, N., L.L. Lao, E.A. Lazarus, M.C. Zarnstorff, J.D. Hanson, S.P. Hirshman, et al., "Plasma Control for NCSX and Development of Equilibrium Reconstruction for Stellarators" at the 2004 IAEA Fusion Energy Conference in Villamoura, Portugal, Nov.1-6, 2004.

18. Phil Snyder, "Study of Toroidal Flow Shear Effects on Intermediate to High-n MHD Stability", Bull. Am. Phys. Soc. 48, 219 (2003).

19.Phil Snyder presented "ELM Size: Thoughts and 3D Nonlinear Simulation Results," at the ITPA Pedestal group meeting, Culham, England, 2 March 2004 ITPA.

20.G. Staebler, "GLF2003 - A Theory Based Transport Model for the Edge and Core”, Bull. Am. Phys. Soc. 48, 261 (2003).

21.G.M. Staebler, "A Gyro-Landau-Fluid Model for Trapped and Circulating Particles" presented at the 2004 Sherwood Conference in Missoula, Montana April 26-28.

22. H. St John, "AT Scenario Modeling for DIII-D and BPX”, Bull. Am. Phys. Soc. 48, 266 (2003).

23. Turnbull, "Equilibrium and Stability for Compact Stellerators", Bull. Am. Phys. Soc. 48, 264 (2003).

24.R. Waltz, "Gyrokinetic Turbulence Simulations With Transport Solutions in GYRO”, Bull. Am. Phys. Soc. 48, 260 (2003).

25.R.E. Waltz, "Gyrokinetic Simulations of Neoclassical Flows Embedded in Turbulence" presented at the 2004 Sherwood Conference in Missoula, Montana April 26-28.

26.S.K. Wong, "Drift Kinetic Formulation of Neoclassical Transport Theory for Plasmas With Large Toroidal Rotations”, Bull. Am. Phys. Soc. 48, 260 (2003). 